\title{
Aplicabilidade da termografia por infravermelho na odontologia: uma revisão de literatura atualizada
}

\author{
Applicability of infrared thermography in \\ dentistry: an updated literature review
}

\section{Susana Silva Soares ${ }^{1}$ (i) Alexandre Melo Karam²}

${ }^{1}$ Autora para correspondência. Faculdade Regional da Bahia (Salvador). Bahia, Brasil. susanasoares_87@yahoo.com.br ${ }^{2}$ Faculdade Regional da Bahia. Salvador, Bahia, Brasil. dr_akaram@hotmail.com

RESUMO | Na saúde, o prognóstico está atrelado a um diagnóstico preciso. No entanto, nos exames de diagnósticos por imagem, muitas vezes a subjetividade dos sinais/sintomas, associada à sobreposição de estruturas anatômicas ou presença de artefatos de imagem, dificulta a acurácia de diagnósticos e tratamentos. Há séculos, é sabido que o excesso de calor ou frio está associado à disfunção ou doença. A termografia por infravermelho consiste em um método de diagnóstico funcional por imagem que permite inferir uma disfunção de um órgão/tecido, através da temperatura. Para tal, baseia-se no registro de diferenças de temperatura corporal e na detecção de padrões de assimetria térmica, em regiões contralaterais do corpo. Diante desse contexto, este trabalho objetiva realizar uma revisão narrativa da literatura atualizada sobre a aplicabilidade da termografia por infravermelho na Odontologia. Para a confecção deste trabalho, foram utilizados artigos que abordaram o uso da termografia infravermelha na Odontologia, no período de 1956 a 2019. No panorama atual, em que princípios como: sustentabilidade, eficácia, promoção de saúde e prevenção de doenças, ditam as diretrizes de saúde mundial, este trabalho corrobora com a concepção de que a inserção do exame termográfico na Odontologia pode favorecer diagnósticos precoces de disfunções/patologias, proporcionar um melhor direcionamento para outros exames mais específicos e contribuir para escolha de terapêuticas mais assertivas. Desta forma, embora a termografia por infravermelho ainda possua uma literatura incipiente e uma confiabilidade questionável, a utilização deste método auxiliar de análise mostra-se bastante promissor na Odontologia e em diversas outras áreas da saúde e do conhecimento.

PALAVRAS-CHAVE: Termografia. Diagnóstico por imagem. Doenças bucais.
ABSTRACT | In health, the prognosis is linked to an accurate diagnosis. However, in a diagnostic imaging exam, often the subjectivity of signs/symptoms, associated with the overlapping of anatomical structures or the presence of image artifacts, hindering the accuracy of diagnoses and procedures. For centuries, has been known that excess of heat or cold has been associated with dysfunction or disease. An infrared term consists of a functional diagnostic method for imaging that allows inferring an organ/ tissue dysfunction using temperature. It is based on recording differences in body temperature and detecting patterns of thermal asymmetry in contralateral regions of the body. In this context, this study aims to perform a narrative review updated about the applicability of infrared thermography in Dentistry. Studies regarding the use of infrared thermography in dentistry from 1956 to 2019 were considered eligible. No current panorama, which principles such as: sustainability, effectiveness, health promotion and disease prevention, perform as world health guidelines, this study corroborates with the conception that an insertion of the thermographic exam in Dentistry that can favor early diagnoses of dysfunctions/pathologies, allowing a better direction for other exams and contributions for the choice of more assertive therapies. Thus, although an infrared term still has an incipient literature and a questionable accurate, the use of this auxiliary method of analysis is very promising in Dentistry and in several other areas of health and knowledge.

KEYWORDS: Thermography. Diagnostic imaging. Mouth diseases. Temporomandibular joint disorders. 


\section{Introdução}

A mensuração da temperatura para investigação de doenças é considerada uma prática milenar, na história da civilização humana. Hipócrates (400 a.C.) postulou em um dos seus aforismos que, onde houvesse excesso de calor ou frio, a doença estaria lá para ser descoberta. Naquela época, Hipócrates e os demais filósofos da Grécia antiga utilizavam a lama como recurso terapêutico para o diagnóstico de doenças, onde a lama secasse mais rápido, ali estava a origem do problema. Este tipo de diagnóstico termobiológico, realizado com lama, foi intitulado pela Dra. Márcia Maria Peixoto Leite, uma das pioneiras da termografia no Brasil, como Termolamal. Com o passar dos anos, o avanço tecnológico permitiu que a avaliação da temperatura corporal humana passasse a ser registrada por termômetros, inicialmente inventado por Galileu Galilei, em 1592, e aperfeiçoado para utilização na saúde em 1612, por Santorio Sanctorius. Entretanto a mensuração da temperatura sem contato só foi permitida graças à descoberta da radiação infravermelha, pelo astrônomo inglês Frederick Willian Herschel, em 1800朋.

Atualmente, o mundo vive uma nova era no diagnóstico térmico. Termógrafos de altíssima resolução e sensibilidade térmica vêm contribuindo para construção de diagnósticos e tratamentos mais assertivos. Por exemplo, neste ano (2020) o mundo foi acometido por uma pandemia viral que causou a morte de milhares de pessoas. A doença COVID-19, como ficou conhecida, causa complicações respiratórias graves em determinados indivíduos, pertencentes a grupos classificados como de risco, e em poucos dias estes pacientes podem evoluir para o óbito. Como, até a presente data, não se tem vacina nem tratamento específico para esta doença, os médicos, cientistas e autoridades mundiais passaram a preconizar a prevenção como melhor aliada. Desta forma, como o aumento da temperatura corporal é um dos principais sinais/sintomas desta doença, termógrafos e termômetros sem contato passaram a ser utilizados em hospitais, aeroportos, empresas e outros estabelecimentos no mundo inteiro.
Na saúde, a termografia por infravermelho é definida como um exame funcional por imagem não ionizante e não invasivo, que mensura à distância a radiação infravermelha emitida pela superfície cutânea, através de um equipamento denominado termógrafo ou câmera termográfica. Desta forma, é possível registrar a distribuição térmica em qualquer região do corpo do paciente. O processo de investigação de disfunções e doenças, através da termografia por infravermelho, baseia-se no princípio de que a microcirculação se altera em face de diferentes condições patológicas朋.

Assim como na Medicina, na Odontologia os achados termográficos potencializam os achados clínicos, proporcionando diagnósticos de alterações fisiopatológicas mais assertivos e, assim, indicar terapias mais apropriadas, talvez até, menos invasivas.

Mesmo diante de grande avanço tecnológico, as dores orofaciais, com toda sua complexidade e subjetividade, ainda hoje, constituem um grande desafio para médicos, dentistas e fisioterapeutas. Entretanto, com a utilização da termografia, é possível determinar, em tempo real, alteração de temperatura na região algógena da dor, nem sempre identificada pelo examinador ou reportada pelo próprio indivíduo胞.

Na Odontologia, além de ajudar na elucidação das dores orofaciais, este método pode auxiliar no diagnóstico de condições como disfunções temporomandibulares (DTM), alterações neuropáticas, doenças periodontais e pulpares

Embora seja notória a necessidade de padronização e um maior rigor metodológico nos estudos para validação da termografia por infravermelho como método diagnóstico na Odontologia, é inegável o papel promissor deste tipo de exame por imagem, no auxílio de diagnóstico e tratamento de patologias e complicações que afetam o sistema estomatognático.

Diante do exposto, este trabalho objetiva realizar uma revisão narrativa da literatura atualizada sobre a aplicabilidade da termografia por infravermelho na Odontologia, e evidenciar o quanto a adoção desta tecnologia pode enriquecer a prática clínica diária do cirurgião-dentista. 


\section{Materiais e métodos}

Este trabalho consiste em uma revisão de literatura qualitativa e descritiva sobre a aplicabilidade da termografia por infravermelho na Odontologia. Para confecção deste trabalho, foi realizada uma busca em bases de dados eletrônicos e websites internacionais, utilizando-se as seguintes palavras-chave: thermography, diagnostic imaging, mouth diseases and temporomandibular joint disorders (termografia, diagnóstico por imagem, doenças bucais e transtornos da articulação temporomandibular).

Foram selecionados manuscritos publicados em inglês e em português, que abordassem o uso da termografia por infravermelho na Odontologia. Os artigos considerados elegíveis compreenderam o período de 1956 a 2019. Trabalhos que não estavam disponíveis na íntegra não foram incluídos na pesquisa.

Foi elaborado um diagrama (figura 1) para mostrar todo o processo de seleção dos artigos. Neste, o número de artigos em cada etapa está representado entre parênteses.

Figura 1. Estratégia de busca dos manuscritos

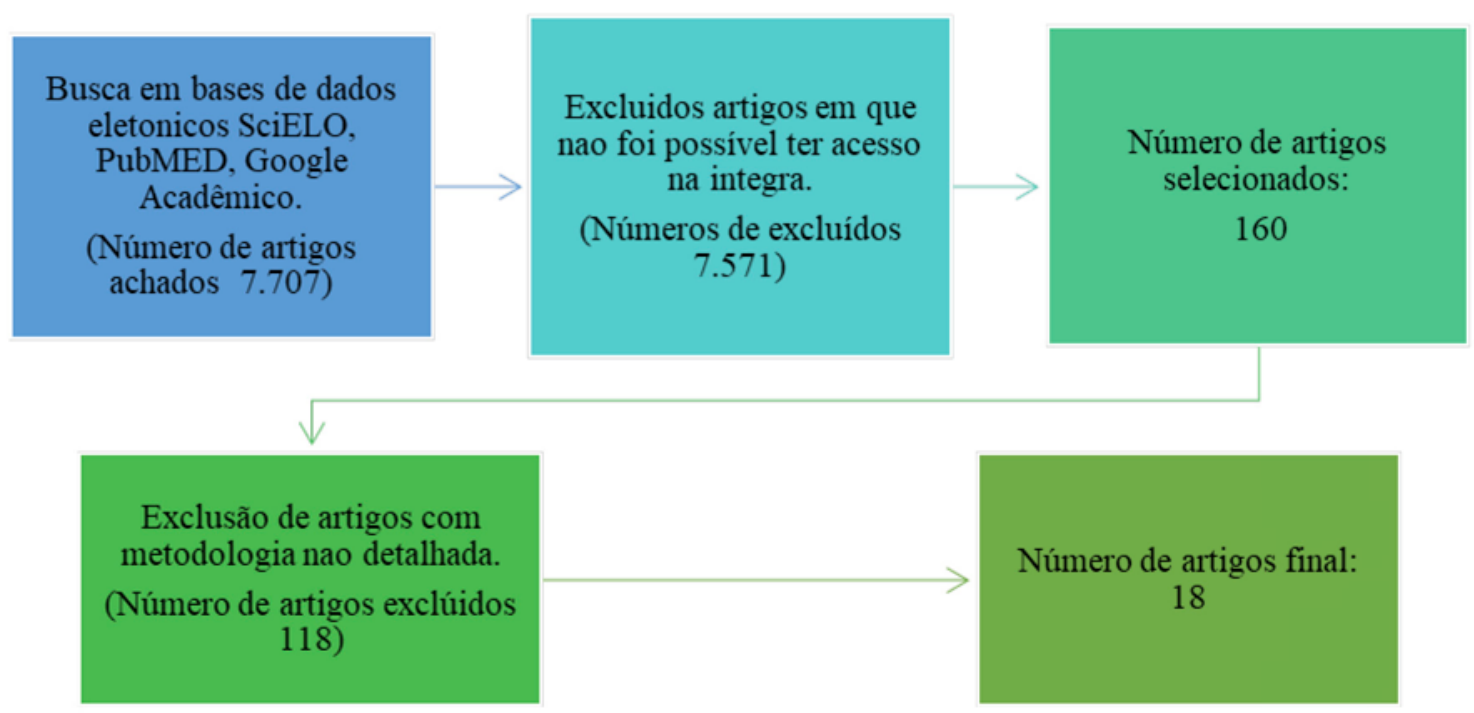

\section{Discussão}

A termografia por infravermelho, também denominada de termometria cutânea infravermelha, termografia médica infravermelha, termografia clínica funcional, consiste em um método de diagnóstico por imagem, não ionizante e não invasivo, que mensura à distância a radiação infravermelha emitida pela superfície cutânea da pele ${ }^{3,14}$.

Este exame capta e registra a distribuição térmica da superfície cutânea dos pacientes com base na microcirculação que se altera em função de diferentes condições patológicas荫. Assim, entende-se que a temperatura exerce um papel muito importante na execução do exame termográfico.

A temperatura da pele é uma função do fluxo sanguíneo, e este, por sua vez, é regulado pelo sistema nervoso vegetativo. De acordo com a literatura, o controle central da temperatura da pele promove uma simetria bilateral dos padrões térmicos do corpo diante de condições de normalidade脚. 
Na saúde, a termografia por infravermelho baseia-se no registro de diferenças de temperatura corporal $(\Delta T)$ e na detecção de padrões de assimetria térmi$c a$, em regiões contralaterais do corpo. $O \Delta T$, também conhecido como parâmetro de assimetria, em relação ao lado contralateral do corpo, consiste no parâmetro mais utilizado na avaliação infravermelha. Desta forma, registros de $\Delta T$ superiores a $0,3^{\circ} \mathrm{C}$ já pode ser indicativo de disfunção em algum órgão ou tecido, demonstrado topograficamente pela imagem termográfica唒阳.

Em 2020, baseando-se nestes princípios, termógrafos passaram a ser utilizados mundialmente, na identificação de possíveis infectados pela COVID-19. Nesta doença, o aumento da temperatura corporal e a dificuldade respiratória são os principais sinais/sintomas. Além do termógrafo identificar alterações na temperatura do indivíduo, este equipamento também é capaz de avaliar, de forma objetiva, o padrão respiratório do indivíduo, visto que a temperatura ao redor das narinas ou da boca oscila durante o ciclo respiratório. Durante a inspiração, a inalação do ar frio do ambiente evidencia a região de interesse analisada mais hiporradiante, enquanto que, durante a expiração o que se observa é uma imagem mais hiperradiante, devido ao ar quente exalado dos pulmões 18 .

Entretanto a implantação desta tecnologia, no serviço de saúde pública e privada, está subutilizada. Além de poder averiguar a temperatura e os padrões respiratórios do paciente, a termografia por infravermelho é capaz de auxiliar e monitorar cirurgias, como a de transplante de órgãos e a de enxertos de pele, processos de cicatrização/reparo tecidual, diferentes tipos de câncer, condições inflamatórias, infecções, disfunções vasculares, lesões neuro-músculo-esqueléticasiged.

Outra contribuição bastante relevante da termografia por infravermelho é no diagnóstico precoce e acompanhamento da osteorradionecrose, uma das mais graves complicações derivadas da radioterapia em região de cabeça e pescoço. O tratamento desta patologia consiste em um grande desafio para a Odontologia e a Medicina. Embora diferentes métodos bioxidativos estejam apresentando resultados bastante vantajosos no tratamento de lesões de osteorradionecrose nos ossos maxilares, a prevenção ainda é vista como o melhor método terapêutico para estas lesões. Em concordância, Jedrusik-Pawłowska et al.21 concluíram que a termografia consiste em um método eficaz no monitoramento da oxigenoterapia hiperbárica, um tipo de terapia bioxidativa, utilizada como tratamento auxiliar em pacientes com osteorradionecrose. Entretanto os autores reforçaram que as imagens termográficas, também chamadas de termogramas, devem ser interpretadas em conjunto com os achados clínicos e radiológicos correspondentes.

Durnovo et al.1. método confiável, altamente informativo, não invasivo, seguro e que não precisa de treinamento técnico. Entretanto, embora os autores defendam que a termografia por infravermelho não demanda um treinamento específico, este estudo corrobora com a percepção de que, para obtenção de um diagnóstico seguro, é necessário que exigências mínimas sejam seguidas, durante a realização do exame termográfico, incluindo a capacitação do profissional que esteja operando a máquina termográfica, para que os termogramas gerados reproduzam com fidedignidade o estado funcional do indivíduo, naquele momento do exame termográfico.

Embora o astrônomo inglês Frederick Willian Herschel tenha descoberto a radiação infravermelha em 1800, somente em 1940 foram desenvolvidos os primeiros sensores capazes de captar a radiação infravermeIha emitida por objetos e transformá-la em imagens térmicas (termogramas). Estes sensores detectam e registram padrões térmicos da superfície da pele do paciente, através de uma câmera infravermelha, também conhecida como termógrafo. Desta forma, a energia térmica emitida pelo corpo humano é captada e convertida em sinais de vídeo eletrônicos, que são então amplificados e transmitidos para um monitor na forma de termogramas. Todo este processo é realizado sem qualquer contato com a superfície alvo. Por fim, software específicos favorecem a análise destas imagens, possibilitando o mapeamento e a medição da temperatura de qualquer região corpórea?.

As primeiras pesquisas utilizando termogramas ocorreram durante a II Guerra Mundial, para rastreamento de tropas inimigas, através dos raios infravermelhos emitidos pelo corpo humano, mesmo no escuro. Alguns anos depois, em 1956, o Canadá compartilhou com o mundo o primeiro registro termográfico na Medicina. Nesse estudo, o Dr. Ray N. Lawson descobriu que a temperatura cutânea de uma mama com

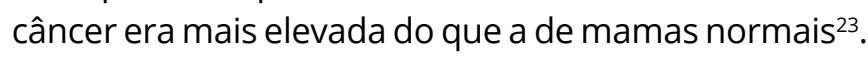


Embora muitos trabalhos na área médica tenham sido impulsionados após a publicação do Dr. Ray, elevando a termografia a um papel de destaque em diversas áreas da saúde, a baixa resolução e sensibilidade térmica dos sensores infravermelhos, até o final da década de 1980, culminaram na publicação de trabalhos controversos e inconclusivos. Atualmente, os termógrafos possuem altíssima resolução e a sensibilidade térmica chega até $0,02^{\circ} \mathrm{C}$, na faixa do infravermelho longo (7-14 $\mu \mathrm{m})$, comprimento de onda que capta aproximadamente $98 \%$ da radiação infravermelha emitida pelo corpo humano (emissividade) 9 . Estas características dos termógrafos mais recentes oferecem maior precisão e segurança para o diagnóstico.

Na Odontologia, a utilização da termografia por infravermelho ainda se mostra bastante incipiente. Entretanto as evidências apontam que este método pode auxiliar o cirurgião-dentista no diagnóstico de condições como disfunção temporomandibular (DTM), dor miofascial, alterações nervosas, odontalgia atípica, processos inflamatórios e infecciosos na região orofacial, como doenças periodontais e pulpares 1 11

Canavan e Gratt da termografia por infravermelho em paciente com DTM, evidenciaram um alto nível de assimetria térmica na região da articulação temporomandibular (ATM), no grupo de pacientes com DTM. Os autores encontraram uma correlação positiva entre gravidade dos sinais e sintomas da DTM e alterações dos valores médios do $\Delta T$ nesta região. Nesse estudo, o aumento de temperatura na região da ATM foi diretamente proporcional ao nível de dor relatado pelos pacientes. Foram encontradas diferenças estatísticas entre os indivíduos sem dor na ATM e os que relataram desconforto leve, moderado e grave à palpação dessa região. Os autores desse estudo concluíram que, quando o diagnóstico não é claro com base nos achados clínicos, a termografia infravermelha pode ser uma ajuda útil no diagnóstico.

Fikackova e Ekberg22, em um estudo de revisão literária sobre o uso da termografia por infravermelho no diagnóstico de artralgia na ATM, relataram que os achados termográficos, descritos na literatura analisada, evidenciam presença assimetria témica em topografia de ATM em pacientes com DTM. Entretanto as autoras concluíram que, para diagnóstico específico de artralgia na ATM, a termografia por infravermelho mostrou ser um método de diagnóstico inconclusivo. Portanto não deve ser recomendado o uso rotineiro da técnica, como um método de diagnóstico isolado para DTM.

Em um estudo sobre o valor da imagem infravermeIha na avaliação da dor, de acordo com alguns autores任 quando a história clínica ou exame físico não são claros, a informação adicional fornecida pela imagem IR anormal pode sugerir um diagnóstico que, até que se prove o contrário, é o correto. Além disto, os autores acrescentam que as imagens infravermelhas complementam as observações anatômicas e estruturais obtidas por outras técnicas radiológicas, como radiografia, mielografia, tomografia computadorizada e ressonância magnética.

Durnovo et al. 1 , em um estudo sobre a aplicação da termografia infravermelha no diagnóstico de diferentes condições maxilofaciais patológicas, utilizando uma amostra de 250 pacientes, observaram que houve um aumento significativo nos índices de temperatura local nas doenças inflamatórias situadas na região de cabeça e pescoço. Por sua vez, no inverso: a diminuição de índices de temperatura foi evidenciada no centro de cistos radiculares e de outras condições crônicas, como osteomielite na fase crônica. De acordo com os achados desse estudo, é possível sugerir uma determinada patologia pelo padrão de temperatura apresentado no termograma.

Em uma revisão sistemática9, avaliando o uso da termografia infravermelha na Odontologia, autores confirmaram a hipótese analisada, de que a termografia por infravermelho é um método auxiliar de diagnóstico na Odontologia, especialmente para os casos que envolvem oscilação térmica derivadas de DTM e aqueles em que há comprometimento dos músculos mastigatórios.

Corroborando com os autores supracitados, Amorim et al. . afirmaram que na Odontologia, a aplicação da termografia é mais notória no campo das DTM. Os autores acrescentam que, além de se apresentar como exame complementar de boa performance na detecção de pontos gatilho da dor, a termografia também pode ser utilizada para acompanhamento de pacientes, de forma não invasiva e como documentação médico-legal. 
Na saúde, a termografia por infravermelho, além de fornecer informações sobre o funcionamento normal e anormal do sistema nervoso simpático e parassimpático, disfunção vascular e trauma miofacial, também é capaz de evidenciar processos inflamatórios localizados朋帽. Embora os estudos na área da Odontologia se concentrem mais na avaliação das

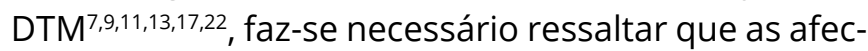
ções pulpares e as doenças periodontais constituem os processos infecto inflamatórios de maior prevalência na cavidade bucal. Desta forma, assim como a termografia tem acurácia na detecção de pontos gatiIho da dor, pode-se inferir que este método também pode ser capaz de identificar assimetrias térmicas no terço inferior da face, as quais podem sugerir um foco dentário ou uma alteração periodontal.

Em seu trabalho, Amorim et al. afirmaram que na Odontologia a termografia pode auxiliar no diagnóstico e planejamento do tratamento de alterações orofaciais. Dentre estas, os autores citaram processos inflamatórios e infecciosos nessa região, certamente referindo-se ao foco dentário ou alteração periodontal.

Assim, mediante dados científicos e observações clínicas vivenciadas, parece ser plausível inferir que, achados termográficos na região peribucal, como presença de hot spots (pequenas áreas de hiperradiação, bem circunscritas) ou hiperradiação difusa na região peribucal, com ou sem drenagem para a região de linfonodos submandibular e sublinguais, podem alertar o cirurgião-dentista quanto à presença de alterações bucais, como abscesso endodôntico ou periodontal, sequestros ósseos em sua fase aguda (osteonecrose, oesteomielite), periodontite, trauma oclusal. Condições estas que, inclusive, podem estar ainda em nível subclínico ou, simplesmente, assintomáticas. Da mesma forma, imagens hiporradiantes na região bucal podem sugerir isquemia/hipóxia, como paralisia facial, ou, como já foi dito anteriormente, lesões císticas ou equestros ósseos em sua fase crônica国.

Além de poder detectar estes processos infecto inflamatórios no sistema estomatognático, a termografia por infravermelho pode fornecer informações objetivas e quantitativas da dor, inclusive a dor orofacial: um tipo de dor extremamente complexa e inconclusivat?.
Na saúde é sabido que o prognóstico está baseado no diagnóstico e nas possibilidades terapêuticas. A precisão térmica e a resolução dos dispositivos termográficos de infravermelho vêm melhorando de acordo com os avanços na tecnologia. A depender do dispositivo utilizado e do propósito que se queira atingir, pode-se ober uma análise quantitativa, através da técnica de termografia, e, assim, fornecer medições precisas de temperatura da superfície alvo, ideal para estudos científicos, ou apenas informações qualitativas, através da técnica de termoscopia, que fornece informações apenas sobre a distribuição de temperatura nessa superfície, excelente para acompanhamento clínico diário do profissional da saúde.

De acordo com Ring e Ammereq, a credibilidade e aceitação da imagem térmica está atrelada ao uso criterioso da tecnologia e ao entendimento adequado da fisiologia térmica. Portanto, para que a Termografia por Infravermelho, independente da técnica utilizada: Termografia e/ou Termoscopia, possa fornecer resultados válidos e, finalmente, ser consolidada como um método auxiliar no diagnóstico, planejamento e monitoramento de intervenções terapêuticas, é necessário reduzir os fatores que podem vir a influenciar os padrões térmicos da superfície alvo. Além disso, é necessário seguir condições-padrão mínimas, para realização de um procedimento termográfico, pois estudos já evidenciaram que, mesmo em indivíduos saudáveis, a temperatura da superfície da pele pode variar ao longo do dia e ser influenciada por vários fatores internos e externos, tais como: exercício físico, utilização de produtos farmacêuticos, condições ambientais e emocionais朋?.

Embora a termografia por infravermelho seja um excelente método auxiliar de diagnóstico, números ainda incipientes de trabalhos científicos e o desconhecimento a respeito do tema na saúde, de um modo geral, limitam o uso da termografia em larga escala na Odontologia e demais áreas da saúde. Além disto, também se observa que as diversas nomenclaturas utilizadas para abordagem do método podem se apresentar como um fator que gera dúvidas para o leitor, no entendimento do assunto. 


\section{Considerações finais}

A termografia por infravermelho é um exame funcional por imagem acessível à pesquisa e à prática clínica diária do cirurgião-dentista, capaz de auxiliar no diagnóstico de diversas alterações presentes no sistema estomatognático. A falta de conhecimento acerca da topografia anatômica e fisiologia térmica limita o exame. Entretanto estudos mostram que na Odontologia, com os termógrafos atuais, já é possível sugerir uma determinada patologia, através do padrão de temperatura observado nas imagens térmicas, com segurança e efetividade.

Trata-se de uma ferramenta de diagnóstico auxiliar viável, e, assim como qualquer outro exame auxiliar, não deve ser utilizada isoladamente. A correlação dos achados termográficos com a avaliação clínica detalhada do paciente e, se necessário, também com outros exames complementares, é imprescindível para obtenção de um diagnóstico e tratamento mais assertivo.

O exame termográfico amplia a visão do profissional da saúde, permitindo-lhe enxergar aspectos fisiológicos invisíveis ao olho humano, e favorece um atendimento mais holístico, integrado e humanizado ao paciente.

Por fim, este trabalho deixa como sugestão que, independente da técnica, Termoscopia ou Termografia, ou do propósito, clínico/científico, o exame termográfico seja mais realizado e explorado pelos profissionais da saúde, haja vista se tratar de uma ferramenta viável e extensiva à propedêutica médica, odontológica, fisioterápica e veterinária.

\section{Contribuições dos autores}

Soares S redigiu o manuscrito e o revisou criticamente. Aprovou a versão final do estudo e acordou em ser responsável por todos os aspectos do trabalho, garantindo que as questões relacionadas à precisão ou integridade de qualquer parte do trabalho fossem investigadas e resolvidas adequadamente. Karam A contribuiu com conteúdo intelectual crítico e revisou a versão final do manuscrito.

\section{Conflitos de interesses}

Nenhum conflito financeiro, legal ou político envolvendo terceiros (governo, empresas e fundações privadas, etc.) foi declarado para nenhum aspecto do trabalho submetido (incluindo, mas não se limitando a subvenções e financiamentos, participação em conselho consultivo, desenho de estudo, preparação de manuscrito, análise estatística, etc.).

\section{Referências}

1. Leite MMP. Comunicação Pessoal. Curso de Pós-Graduação em Termologia Aplicada à Saúde e Termografia Clínica Funcional. Instituto de Desenvolvimento Educacional. [Internet]. 2019. Disponível em: https://www.faculdadeide.edu.br/pos-graduacao/ termologia-saude-termografia-clinica-funcional-salvador/

2. Anbar M, Gratt BM, Hong D. Thermology and facial telethermography. Part I: history and technical review. Dentomaxillofac Radiol. 1998;27(2):61-7. doi: 10.1038/sj/ dmfr/4600314

3. Brioschi ML, Teixeira MJ, Silva MF. Princípios e Indicações da Termografia Médica. 1.ed. São Paulo: Andreoli; 2010.

4. Brioschi ML, Macedo JF, Macedo RAC. Termometria Cutânea Infravermelha de Alta Sensibilidade (T.I.A.S.) - Definição, Aplicações e Especificações. Rev Méd Paraná. 2001;59(2):56-63.

5. Vargas JVC, Brioschi ML, Dias FG, Parolin MB, Mulinari-Brenner FA, Ordonez JC et al. Normalized methodology for medical infrared imaging. Infrared Physics \& Technology. 2009;52(1):42-47. doi: 10.1016/j.infrared.2008.11.003

6. Haddad DS, Brioschi ML, Baladi MG, Arita ES. A new evaluation of heat distribution on facial skin surface by infrared thermography. Dentomaxillofac Radiol. 2016;45(4):20150264. doi: 10.1259/dmfr.20150264

7. Amorim AMAM, Barbosa JS, Freitas APLF, Viana JEF, Vieira LEM, Suassuna FCM et al. Termografia infravermelha na Odontologia. HU Revista. 2018;44(1):15-22. doi: 10.34019/1982-8047.2018. $\underline{\mathrm{v} 44.13943}$

8. Canavan D, Gratt BM. Electronic thermography for assessment of mild and moderate temporomandibular joint dysfunction. Oral Surg Oral Med Oral Pathol Oral Radiol Endod. 1995;79(6):778-86. doi: 10.1016/s1079-2104(05)80316-6

9. Presídio LR, Wanderley FGC, Medrado ARAP. O uso da termografia infravermelha na odontologia e suas especialidades: uma revisão sistemática. Rev Bahiana Odonto. 2016;7(2):155-165. doi: $10.17267 / 2238-2720$ 
10. Aires Júnior FAF. Otimização do processo metodológico para aquisição de imagens termográficas da face [tese]. Campina Grande: Universidade Federal de Campina Grande; 2018.

11. Manfredini D, Guarda-Nardini L, Winocur E, Piccotti F, Ahlberg J, Lobbezoo F. Research diagnostic criteria for temporomandibular disorders: a systematic review of axis I epidemiologic findings. Oral Surg Oral Med Oral Pathol Oral Radio. Endod. 2011;112(4):453-62. doi: 10.1016/j.tripleo.2011.04.021

12. Durnovo EA, Potekhina YP, Marochkina MS, Yanova NA, Sahakyan MY, Ryzhevsky DV. Diagnostic Capabilities of Infrared Thermography in the Examination of Patients with Diseases of Maxillofacial Area. CTM. 2014;6(2): 61-65.

13. Melo DP, Bento PM, Peixoto LR, Martins SKLD, Martins $C C$. Is infrared thermography effective in the diagnosis of temporomandibular disorders? A systematic review. Oral Surg Oral Med Oral Pathol Oral Radiol. 2019;127(2):185-192. doi: 10.1016/j.0000.2018.09.006

14. Brioschi ML, Macedo JF, Macedo RAC. Skin thermometry: new concepts. J Vasc Br. 2003;2(2):151-60.

15. Gratt BM, Sickles EA. Electronic facial thermography: an analysis of asymptomatic adult subjects. J Orofac Pain. 1995;9(3):255-65.

16. Brioschi ML, Abramavicus S, Corrêa CF. Valor da imagem infravermelha na avaliação da dor. Rev Dor. 2005;6(1):514-524.

17. Haddad DS, Brioschi ML, Vardasca R, Weber M, Crosato EM, Arita ES. Thermographic characterization of masticatory muscle regions in volunteers with and without myogenous temporomandibular disorder: preliminary results. Dentomaxillofac Radiol. 2014;43(8):1-7. doi: 10.1259/ dmfr. 20130440

18. Telson YC, Motta AR, Furlan RMMM, Estevam P, Porto MP, Huebner $\mathrm{R}$ et al. Potencial de aplicação da termografia na avaliação do modo respiratório. In: ENEBI 2018 - $6^{\circ}$ Encontro Nacional de EngenhariaBiomecânica. 2018; Água de Lindóia, São Paulo; 2018. doi: 10.26678/ABCM.ENEBI2018.EEB18-0038

19. Matias JEF, Morais FA, Kato DMP, Koziak V, Brioschi ML, Tambara EM et al. Prevenção de isquemia hepática normotérmica durante perfusão hepática in situ com três diferentes soluções de preservação: análise experimental através de termografia por radiação infravermelha em tempo real. Rev Col Bras Cir. 2010;37(3):211-217. doi: 10.1590/S0100-69912010000300009

20. Ring EFJ, Ammer K. Infrared thermal imaging in medicine. Physiol Meas. 2012;33(3):33-46. doi: 10.1088/0967-3334/33/3/R33

21. Jedrusik-Pawłowska M, Niedzielska I , Bogucki R, Kajewski B. Effectiveness of hyperbaric oxygen therapy in mandibular osteoradionecrosis shown by thermography monitoring. Med Sci Monit. 2010;16(2):MT1-8.
22. Fikackova H, Ekberg EC. Can infrared thermography be a diagnostic tool for arthralgia of the temporomandibular joint? Oral Surg Oral Med Oral Pathol Oral Radiol Endod 2004;98(6):64350. doi: 10.1016/j.tripleo.2004.02.080

23. Lawson RN. Implications of surface temperatures in the diagnosis of breast cancer. Can Med Assoc J. 1956;75(4):309-310. 